Acta Protozool. (2020) 59: 55-60

www.ejournals.eu/Acta-Protozoologica

doi:10.4467/16890027AP.20.004.12160

PROTOZOOLOGICA

Short communication

\title{
Tolerance of Colpoda cucullus Nag-1 Resting Cysts and Presumed Structure for Protection against UV Light
}

\author{
Shinji YAMANE ${ }^{1}$, Maho WATANABE ${ }^{1}$, Ryoji FUNADANI ${ }^{1}$, Ryutaro MIYAZAKI ${ }^{1}$, Yuya \\ HASEGAWA ${ }^{1}$, Mikihiko ARIKAWA ${ }^{2}$, Futoshi SUIZU ${ }^{3}$, Kou MATSUOKA ${ }^{4}$, Tatsuomi MATSUOKA ${ }^{2}$ \\ ${ }^{1}$ Department of Biological Science, Faculty of Science, Kochi University, Kochi 780-8520, Japan \\ ${ }^{2}$ Department of Biological Science, Faculty of Science and Technology, Kochi University, Kochi 780-8520, Japan \\ ${ }^{3}$ Division of Cancer Biology, Institute for Genetic Medicine, Hokkaido University, Sapporo 060-0815, Japan \\ ${ }^{4}$ Kyowakaibyoin (Kyowakai Hospital), Suita 564-0001, Japan
}

\begin{abstract}
Resting cysts of the terrestrial ciliate Colpoda cucullus (Nag-1 strain) are highly resistant to UV light. It has been speculated that auto-fluorescent (blue fluorescent) particles surrounding the nuclei and yellowish fluorescent layers of the cyst wall are the candidate structures for the protection of the cellular components from UV light. The UV resistance of encysting cells was quickly acquired up to $5 \mathrm{~h}$ after the onset of encystment induction, and then gradually increased for several days. The less fluorescent ectocyst layer, yellowish fluorescent first-synthesized endocyst layer (en-1) and the NSPs were formed within $5 \mathrm{~h}$ after the onset of encystment induction, and thereafter endocyst layers became gradually thicker for several days. The cyst wall sample (ectocyst and endocyst layers) markedly absorbed a broad range of UV light. This result indicates that the cyst wall evidently has UV-cut function. These results support that the cyst wall and NSPs of C. cucullus play a role in the shielding of the cell components from UV light.
\end{abstract}

Keywords: cyst wall, endocyst layer, nuclei-surrounding particles, UV resistance.

\section{INTRODUCTION}

The terrestrial ciliates such as Colpoda transform into resting cysts that are tolerant to desiccation (Corliss and Esser 1974), freezing (Taylor and Strickland 1936, Maeda et al. 2005, Müller et al. 2010), high temperature (Taylor and Strickland 1936, Maeda et al. 2005), hydrochloric acid ( $\mathrm{HCl})$ (Sogame et al. 2011), ultraviolet

Address for correspondence: Tatsuomi Matsuoka: Department of Biological Science, Faculty of Science and Technology, Kochi University, Kochi 780-8520, Japan; Fax: +81-88 844 8356; E-mail: tmatsuok@kochi-u.ac.jp
(UV) rays (Matsuoka et al. 2017), and gamma rays (Sogame et al. 2019) before the water puddles in which the vegetative cells grow dry out. In encystment-induced C. cucullus Nag-1, the digestion of vegetative cell structures by autophagy, a stop of mitochondrial activity (Sogame et al. 2014) and the cell cycle, and the cyst wall formation begin simultaneously at $2-3 \mathrm{~h}$ after the onset of encystment induction. At 2-3 h after the encystment induction, mucus is expelled to form a sticky mucus layer surrounding the cell, followed by an extrusion of sticky small globules called 'lepidosomes' (Foissner et al. 2011), which are trapped by the mucus layer. Thereafter, a rigid ectocyst layer begins to form 
just over the plasma membrane. It may take $3-4 \mathrm{~h}$ to complete a thicker ectocyst layer. A first endocyst layer (en-1) is formed in 3-5 h between the ectocyst layer and plasma membrane, followed by the formation of several endocyst layers over several days. Thus, in the mature resting cyst, the cell is surrounded by a cyst wall composed of a mucous/lepidosome layer, an ectocyst layer, and endocyst layers (from the outermost layer) (Funatani et al. 2010). Mitochondria aggregate in the peripheral region, and nuclei located in the center are surrounded by nuclei-surrounding particles (NSPs) (Matsuoka et al. 2017).

Resistance to UV rays seems to be one of the essential adaptive strategies for inhabitants of the soil surface to survive. We recently observed that the mature resting cysts of $C$. cucullus Nag-1 showed strong resistance to UV light irradiation (the presumed UV dose required for $99.9 \%$ inactivation was $571 \mathrm{~mJ} / \mathrm{cm}^{2}$ ) (Matsuoka et al. 2017) that is 14-fold higher than the resistance of vegetative cells of Colpoda, 11-fold higher than the resistance of Bacillus spores (Nicholson and Galeano 2003), and 4-fold higher than the resistance of soil amoeba Acanthamoeba cysts (Lonnen et al. 2014). We proposed that the yellowish autofluorescent cyst wall and blue-autofluorescent NSPs under the excitation of UV light (Matsuoka et al. 2017) may be involved in UV tolerance. If these fluorescent structures have a role in the protection of cellular components including nuclei from UV light, the formation of these fluorescent structures would be expected to be accompanied by the acquisition of UV tolerance. Therefore, in the present study, we compared the time course of the acquisition of UV tolerance with the formation of auto-fluorescence structures of encysting cells, and demonstrated that cyst wall layers actually absorbed UV rays.

\section{MATERIALS AND METHODS}

\section{Cell culture and the preparation of wet encysting cells and wet mature resting cysts}

Colpoda cucullus Nag-1 strain (Funadani et al. 2016) (18S ribosomal RNA gene: GenBank accession no. AB918716) was cultured in a $0.05 \%(\mathrm{w} / \mathrm{v})$ infusion of dried wheat leaves. Vegetative cells collected by centrifugation $(1500 \times \mathrm{g}$ for $2 \mathrm{~min})$ were suspended in an encystment-inducing solution containing $1 \mathrm{mM}$ Tris- $\mathrm{HCl}(\mathrm{pH}$ 7.2) and $0.1 \mathrm{mM} \mathrm{CaCl}_{2}$, and $200 \mu \mathrm{l}$ of cell suspension containing 50 cells was dispended in a watch glass. A single lot of 50-cell samples of vegetative cells was prepared and maintained in order to encyst under humid conditions.

\section{Preparation of dry resting cysts}

The 2-day cultured vegetative cells were concentrated by centrifugation $(1500 \times \mathrm{g}$ for $2 \mathrm{~min})$, were dispended in a watch glass (at approx. 500 cells per $500-\mu 1$ suspension), and the lot of 500 -cell samples were maintained for 2 weeks to naturally encyst in culture medium under humid conditions (the resting cysts were adhered to the bottom of the watch glass). Thereafter, the medium (old culture medium) was discarded, rinsed once with pure water, and then dried over a long time under semi-humid conditions.

\section{UV irradiation to the resting cysts}

The resting cysts in the watch glass were irradiated with UV light (254-nm light) using a UV sterilizer (Trybest EG-0003). The intensity of the UV light was measured using a UV photometer (MatherTool, SP-82UV). The dose of UV light was adjusted by changing irradiation time. After UV light irradiation was administered to the wet resting cysts, the medium in the watch glass was discarded, and then an infusion of fresh $0.05 \%$ wheat leaves was poured to induce excystment. For the dry resting cysts, a fresh $0.05 \%$ wheat leaves infusion was poured into the cyst-adhered watch glass which had been exposed to UV light.

In the wet cysts, the number of excysted vegetative cells and the number of vacant cysts were counted at $5 \mathrm{~h}$ after the onset of excystment induction by the addition of fresh $0.05 \%$ wheat leaves infusion. For the dry cysts, the resting cysts were randomly chosen and the number of vacant cysts from which vegetative cells had emerged was counted at $12 \mathrm{~h}$ after the onset of excystment induction. The rate of excystment is expressed as the percentage of the total number of observed cells (50 wet cysts: 100-300 dry cysts).

\section{UV light irradiation to vegetative cells}

Vegetative cells collected by centrifugation $(1500 \times \mathrm{g}$ for $2 \mathrm{~min}$ ) were suspended in $1 \mathrm{mM}$ Tris- $\mathrm{HCl}(\mathrm{pH} 7.2)$, and $200 \mu \mathrm{l}$ of cell suspension containing 50 cells was dispended in a watch glass. The number of cells were counted while sucking with a thin pipette. After UV light irradiation using a UV sterilizer (Trybest EG0003), deformed and immobile cells were judged as inactivated cells. In order to confirm that the immobile rounded cells attached to the bottom of a watch glass were not encysting cells, the medium was discarded and a fresh $0.05 \%$ infusion of dried wheat leaves was poured to induce excystment, and then observed in $24 \mathrm{~h}$ after onset of excystment induction.

\section{Electron microscopy and fluorescence microscopy}

The electron microscopy and fluorescence microscopy examinations were performed as described (Funatani et al. 2010, Matsuoka et al. 2017).

\section{Isolation of the cyst wall and its absorption spec- trum}

Vegetative cells of C. cucullus Nag-1 were induced to encyst in a petri dish and maintained for 1 week. The resting cysts were adhered to the bottom of the petri dish. The medium in the petri dish was discarded, and an excystment-inducing medium [a $0.2 \%$ $(\mathrm{w} / \mathrm{v})$ infusion of dried wheat leaves] was poured into the dish. The vegetative motile cells emerged in most resting cysts within $7 \mathrm{~h}$. Therefore, an empty cyst sample (i.e., cyst wall sample) was easily 
obtained by discarding the medium containing emerged vegetative cells at $7 \mathrm{~h}$ after encystment induction. The vacant cysts that had adhered to the bottom of the petri dish were washed three times with water and then the vacant cysts were scraped off the dish with a cutter blade and collected by centrifugation $(8,000 \times \mathrm{g}$ for $10 \mathrm{~min})$. A $20-\mu 1$ pellet was suspended in $700 \mu 1$ of $0.2 \%$ agarose for electrophoresis of DNA and RNA (for $\geq 1 \mathrm{kbp}$ fragment) (Nacalai tesque).

The absorption spectrum of the vacant cyst suspension was determined using a JASCO V-550DS spectrophotometer equipped with a JASCO ISV-469 integrating sphere to minimize the loss of spectral resolution due to scattering. The absorbance of agarose was deducted from the absorbance of agarose containing cyst wall suspension.

\section{RESULTS AND DISCUSSION}

Figure 1 provides Nomarski images of wet resting cysts of $C$. cucullus Nag-1 that had aged $>2$ weeks and their fluorescence photomicrographs obtained by UV excitation, showing blue-fluorescent nuclei-surrounding particles (NSPs) and yellowish fluorescent cyst wall. Electron microscopy (Matsuoka et al. 2017) had shown that the nuclei of the resting cysts were surrounded by NSPs but were not surrounded in the vegetative Colpoda cells. The electron micrographs of the macronucleus surrounded by the NSPs and a magnified image of the mature resting cysts are shown in Fig. 1E, F. The NSPs are electron-dense particles with an electron-lucent core. As shown in Fig. 1C, D, some resting cysts were surrounded by a double-layer of cyst wall emitting yellowish fluorescence. The inner layer was evidently an endocyst, and the outer layer may be composed of an ectocyst layer (ec) and a first-synthesized endocyst layer (en-1), because the first endocyst layer was sticking to the ectocyst layer (Fig. 1G).

Fig. 2 shows the formation of auto-fluorescent structures in encysting cells. At $3 \mathrm{~h}$ after the onset of encystment induction, the cells were rounded and surrounded by an ectocyst layer (ec) covered with a mucous/lepidosome layer (Fig. 2A). The lepidosome ('le') was green or yellowish-fluorescent, and the mucous mass ('mu') was blue-fluorescent. In the encysting cell whose mucous/lepidosome layer had been removed by pipetting, the ectocyst layer was observed to be less fluorescence (Fig. 2B). At this stage, large particles (the presumed precursors of NSPs) began to accumulate in the vicinity of the macronucleus ('ma').

At $5 \mathrm{~h}$, most of the encysting cells were covered with a thin layer emitting yellowish fluorescence (Fig. 2C). The fluorescent thin layer may be a first-synthesized endocyst layer ('en-1'), because at this stage, a first- synthesized endocyst layer was formed in most of the cells (Sogame et al. 2011). At this stage, the NSP formation has almost completed (Fig. 2C). At $10 \mathrm{~h}$, the yellowish fluorescent layer became thicker, and it was difficult to see the NPSs (Fig. 2D). Presumably, a second-synthesized endocyst layer may have been formed. Thereafter, the yellowish fluorescent layer became much thicker (Fig. 2E, F).

We irradiated wet encysting cells of $C$. cucullus Nag-1 at different stages (Fig. 3A) and dry mature cysts (Fig. 3B) with UV light (254 nm), and we plotted the excystment rates (\%) against the UV light value (254 $\mathrm{nm}$ ) (Fig. 3A, B). The degree of UV tolerance (i.e., the UV value necessary for $90 \%$ inactivation) of the wet cysts is shown in the inset of Fig. 3A. The UV tolerance of the wet encysting cells was quickly acquired up to $5 \mathrm{~h}$ after the onset of encystment induction, and thereafter it gradually increased for several days (Fig. $3 \mathrm{~A}$, inset). These results suggest that both the cyst wall and the NSPs may be involved in the protection of cell components from UV light.

In the previous study (Matsuoka et al. 2017), lepidosomes and an ectocyst layer are thought to emit fluorescence. In the present study, we observed that the lepidosomes and endocyst layers were green- or yellowish-fluorescent, and it appeared that the ectocyst layer seemed to be less fluorescence, not yellowish fluorescence (Fig. 2B).

Dry mature cysts (aged $>2$ weeks) were highly resistant to UV light (Fig. 3B). In this case, the presumed UV level required for $99.9 \%$ inactivation was $600 \mathrm{~mJ} /$ $\mathrm{cm}^{2}$, a value which was higher than that $\left(571 \mathrm{~mJ} / \mathrm{cm}^{2}\right)$ of 1-week-aged wet resting cysts (Matsuoka et al. 2017). The UV level required for $99.9 \%$ inactivation is $50 \mathrm{~mJ} /$ $\mathrm{cm}^{2}$ in Bacillus spores (Nicholson and Galeano 2003) and $130 \mathrm{~mJ} / \mathrm{cm}^{2}$ in soil amoeba Acanthamoeba cysts (Lonnen et al. 2014). Our present findings demonstrated that the resting cysts of C. cucullus Nag-1 were more resistant to UV light than other species. The cells of $C$. cucullus Nag-1 aggregate to form resting cysts. Therefore, some of the resting cysts may have been shaded from UV irradiation by other cysts. As a result, the survival rate of UV-exposed cells may be slightly higher.

The results we obtained herein suggest that the cyst wall and NSPs have roles in the protection from UV. The question thus arises: does the cyst wall actually absorb UV light? We collected vacant cyst wall (Fig. 4A) and determined its absorption spectrum by using a spectrophotometer equipped with an integrating sphere to minimize the loss of spectral resolution 
due to scattering (Fig. 4B). In this sample, almost all cysts were vacant. When the vegetative cells had just emerged from the cysts, they were surrounded by fragile yellowish fluorescent thin layers (Funadani et al. 2016), which may be a part of the endocyst layers. A previous electron microscopy examination of some exuvia (vacant cysts) showed that an ectocyst layer of exuvia was not lined with endocyst layers (Funadani et al. 2016). However, the ectocyst layer of the exuvia shown in Fig. 4A-2 seems to be lined with endocyst layers, because the layer emitted a yellowish fluorescence. As shown in Fig. 4B, the cyst wall sample (Fig. 4A) markedly absorbed a broad range of UV light. This result indicates that the cyst wall evidently has UV-cut function.
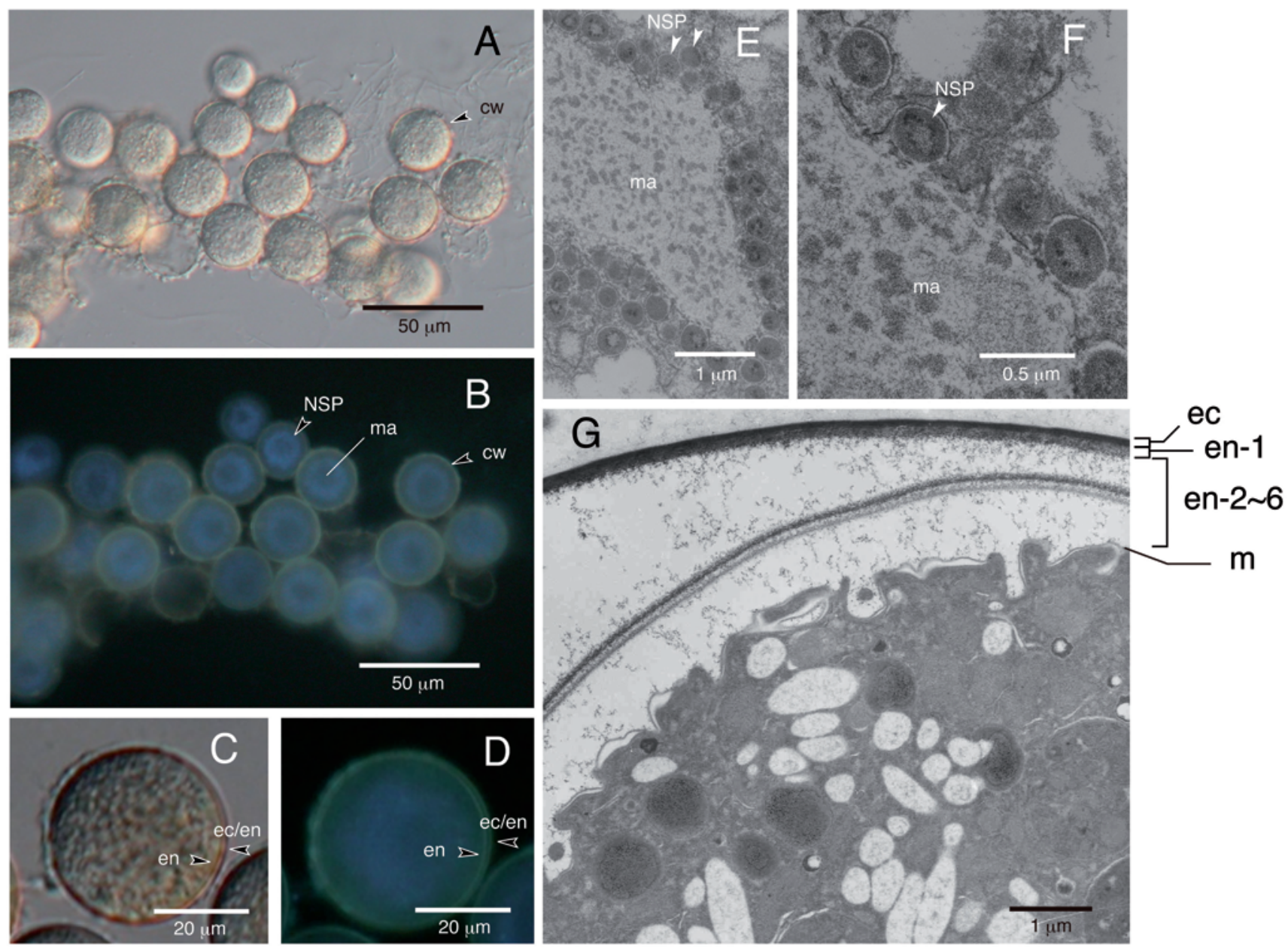

Fig. 1. Photomicrographs of yellowish auto-fluorescent structures of the mature cysts (wet cysts) of C. cucullus Nag-1 aged $>2$ weeks by UV excitation (A-D) and electron micrographs showing auto-fluorescent nuclei-surrounding particles (NSPs) (E, F) and cyst wall components (G). A, B - A Nomarski image (A) of a mature resting cyst and its fluorescence photomicrograph (B) showing autofluorescent structures. cw: cyst wall, ma: macronucleus. C-D - A Nomarski image (C) of a mature resting cyst and its fluorescence photomicrograph (D) showing autofluorescent layers of cyst wall. ec/en: an ectocyst-endocyst layer complex, en: endocyst layer. E-F - Electron micrographs showing NSPs. G - Electron micrograph showing cyst wall components. ec: ectocyst layer, en-1: first-synthesized layer of endocyst, en-2-6: second - sixth layers of endocyst, m: plasma membrane.

Fig. 3. Inactivation of encysting cells (wet cells) (cyst age: 0-10 days) of C. cucullus Nag-1 (A) and mature dry cysts (cyst age $>2$ weeks) (B) by irradiation with UV light $(254 \mathrm{~nm})$. The points were the result of one measurement. Fig. 3A inset - The UV values required for the $90 \%$ inactivation (degree of UV tolerance) of wet cysts, whose values were obtained from Fig. 1A, are plotted against cyst ages. 

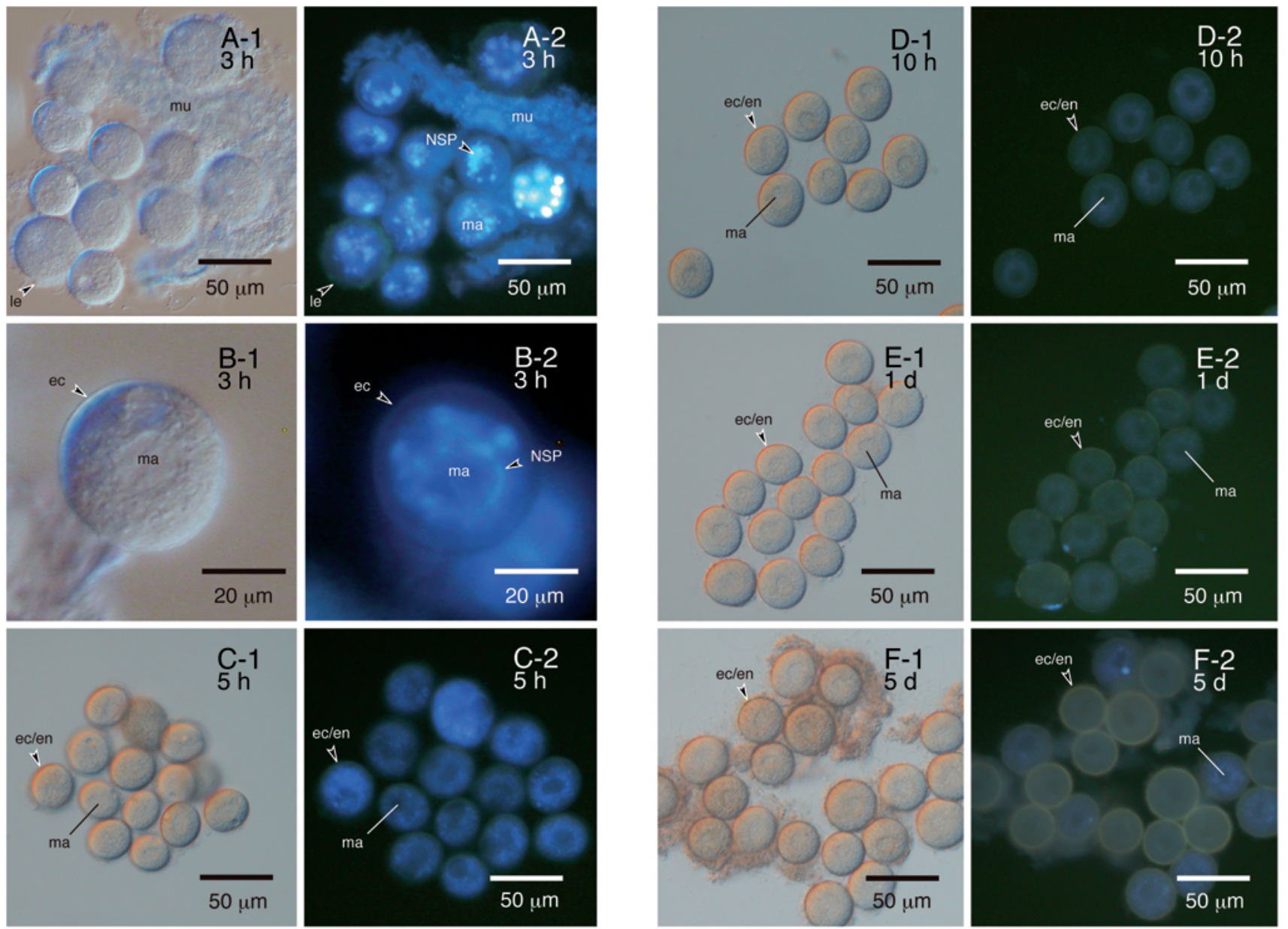

Fig. 2. Nomarski images (A-1-F-1) and their fluorescence photomicrographs (A-2-F-2) showing the formation of the auto-fluorescent cyst wall and NSPs in the encysting cells (wet cysts) after the onset of encystment induction. The cyst age ( $3 \mathrm{~h}$ to 5 days; A-F) is given at the upper right of each photograph. ec/en: ectocyst-endocyst complex, ec: an ectocyst layer, le: lepidosomes, ma: macronucleus, mu: mucus, NSP: nuclei-surrounding particle.
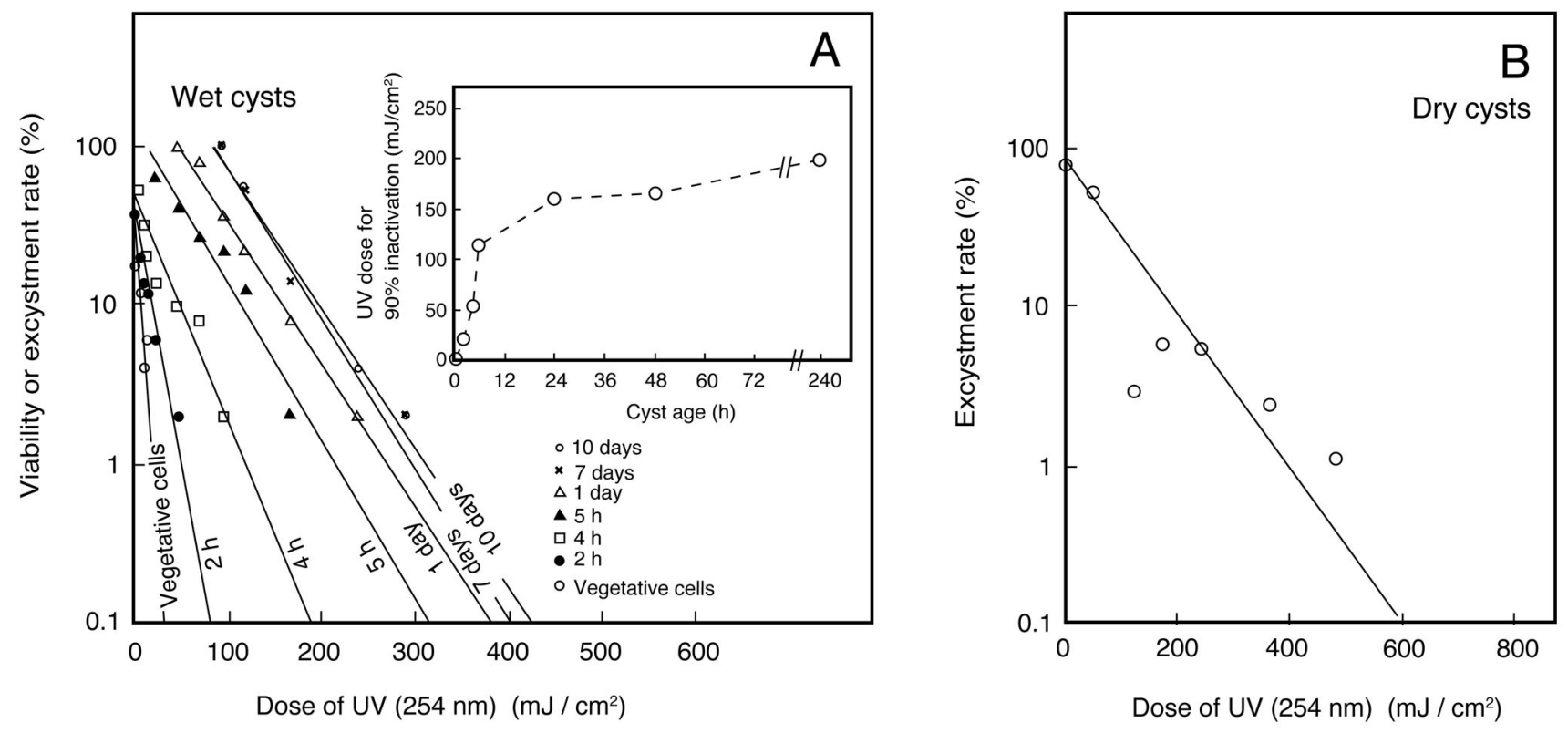

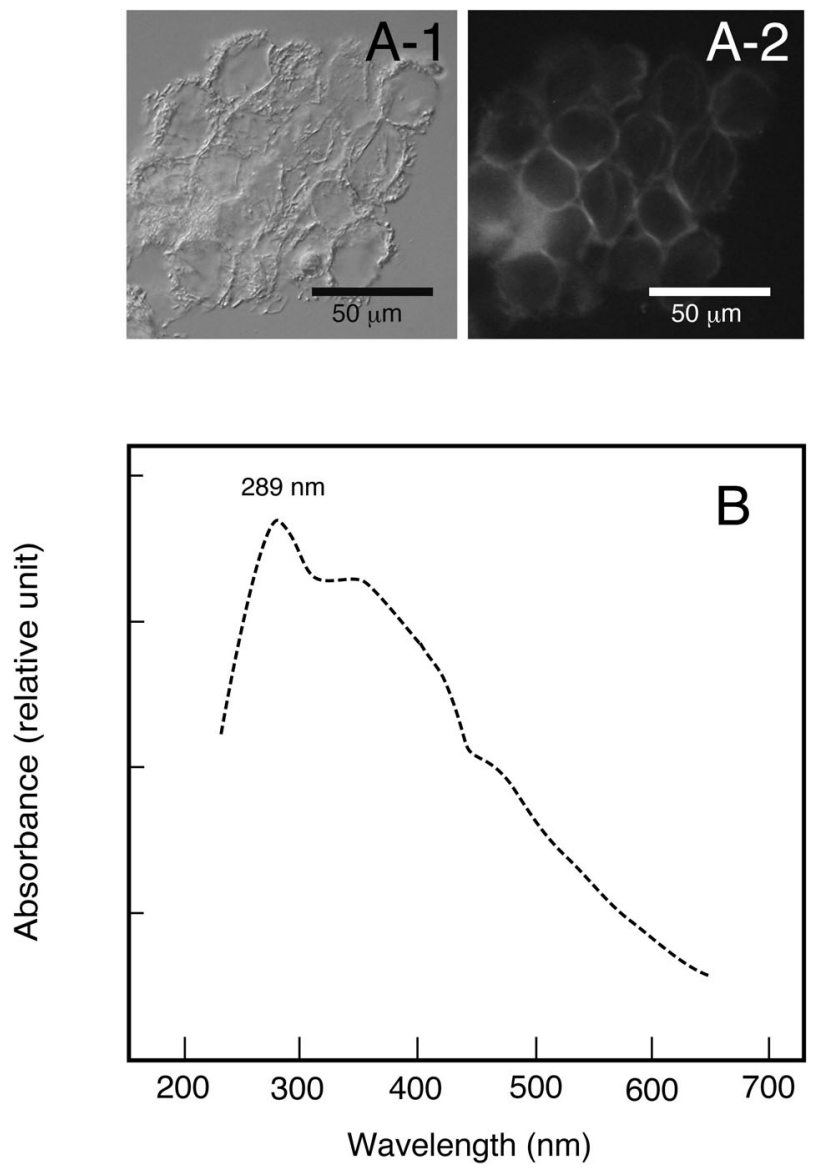

Fig. 4. Nomarski image (A-1) and its fluorescence photomicrograph (A-2) of a vacant cyst (cyst wall sample) obtained from 1-weekaged mature cysts of $C$. cucullus Nag-1, and its absorption spectrum (B) obtained by a spectrophotometer equipped with an integrating sphere.

In excystment-induced Colpoda cucullus Nag-1 cysts, the UV tolerance was maintained just until the vegetative cells emerged from the resting cysts despite the dispersion of the NSPs occurred in most of the cells (data not shown). Judging from this result, the cyst wall seems to play the most important role in the protection of the cell from UV light exposure. The function of the NSPs is probably in the avoidance of an injury of DNA by UV light exposure.

Acknowledgment. The electron micrographs were kindly provided by Dr. Akemi Kida. This research was financially supported by a Grant-in-Aid for Scientific Research (B) (\#19H03447).

\section{REFERENCES}

Corliss J. O., Esser S. C. (1974) Comments on the role of the cyst in the life cycle and survival of free-living protozoa. Trans. Amer. Micros. Soc. 93: 578-593

Foissner W., Stoeck T., Agatha S., Dunthorn M. (2011) Intraclass evolution and classification of the Colpodea (Ciliophora). J. Eukaryot. Microbiol. 58: 397-415

Funadani R., Sogame Y., Kojima K., Takeshita T., Yamamoto K., Tsujizono T., Suizu F., Miyata S., Yagyu K., Suzuki T., Arikawa M., Matsuoka T. (2016) Morphogenetic and molecular analyses of cyst wall components in the ciliated protozoan Colpoda cucullus Nag-1. FEMS Microbiol. Lett. 363: fnw 203

Funatani R., Kida A., Watoh T., Matsuoka T. (2010) Morphological events during resting cyst formation (encystment) in the ciliated protozoan Colpoda cucullus. Protistology 6: 204-217

Lonnen J., Putt K. S., Kernick E. R., Lakkis C., May L., Pugh R. B. (2014) The efficacy of Acanthamoeba cyst kill and effects upon contact lenses of a novel ultraviolet lens disinfection system. Am. J. Ophthalmol. 158: 460-468

Maeda H., Akematsu T., Fukui R., Matsuoka T. (2005) Studies on the resting cyst of ciliated protozoan Colpoda cucullus: resistance to temperature and additional inducing factors for en-or excystment. J. Protozool. Res. 15: 7-13

Matsuoka K., Funadani R., Matsuoka T. (2017) Tolerance of Colpoda cucullus resting cysts to ultraviolet irradiation. J. Protozool. Res. 27: 1-7

Müller H., Achilles-Day U. E. M., Day J. G. (2010) Tolerance of the resting cysts of Colpoda inflata (Ciliophora, Colpodea) and Meseres corlissi (Ciliophora, Spirotrichea) to desiccation and freezing. Eur. J. Protistol. 46: 133-142

Nicholson W. L., Galeano B. (2003) UV resistance of Bacillus anthracis spores revisited: Validation of Bacillus subtilis spores as UV surrogates for spores of B. anthracis Sterne. Appl. Environ. Microb. 69: 1327-1330

Sogame Y., Kida A., Matsuoka T. (2011) Possible involvement of endocyst in tolerance of the resting cyst of Colpoda cucullus against HCl. Afr. J. Microbiol. Res. 5: 4316-4320

Sogame Y., Kojima K., Takeshita T., Kinoshita E., Matsuoka T. (2014) Identification of differentially expressed water-insoluble proteins in the encystment process of Colpoda cucullus by twodimensional electrophoresis and LC-MS/MS analysis. J. Eukaryot. Microbiol. 61: 51-60

Sogame Y., Saito R., Koizumi R., Shimizu T., Ono T. (2019) Evidence of stress recovery in free-living ciliate Colpoda cucullus: The repair capability of resting cysts to damage caused by gamma irradiation. Acta Protozool. 58: 25-29

Taylor C. V., Strickland A. G. R. (1936) Effects of high vacua and extreme temperatures on the cysts of Colpoda cucullus. Physiol. Zool. 9: 15-26

Received on $18^{\text {th }}$ December, 2019; revised on $2^{\text {nd }}$ March, 2020; accepted on $9^{\text {th }}$ March, 2020 